\title{
Comparison of COVID-19 Case-Fatality-Rates by Socio- Demographic Factors
}

\author{
(1) Esra Keleş Peker1, (10) Güven Bektemur2, (1) Kürşad Nuri Baydili3, (1) Melek Aktaş4 \\ 1 University of Health Sciences Turkey, Zeynep Kamil Training and Research Hospital, Clinic of Gynecologic Oncology, İstanbul, Turkey \\ 2University of Health Sciences Turkey, Hamidiye Faculty of Medicine, Department of Public Health, İstanbul, Turkey \\ ${ }^{3}$ University of Health Sciences Turkey, Hamidiye Faculty of Medicine, Department Biostatistics and Medical Informatics, İstanbul, Turkey \\ ${ }^{4}$ French School of Public Health and Political Sciences Rennes, Student master 2 in Administration of Public Health Policies and Programmes, \\ Rennes, France
}

Cite this article as: Keleş Peker E, Bektemur G, Baydili KN, Aktaş M. Comparison of COVID-19 Case-Fatality-Rates by Socio-Demographic Factors. J Acad Res Med 2020;10(3):246-51

\begin{abstract}
Objective: To evaluate socio-demographic risk factors in comparison with Coronavirus disease (COVID-19) case fatality rates (CFRs).

Methods: In this cross-sectional study, we used the demographic and epidemiologic data that were identified as risk factors for COVID-19 CFRs. The electronic dataset was extracted from an open-source database, Our World in Data, and the European Center for Disease Prevention and Control websites. Countries with at least 1,000 confirmed COVID-19 cases that were reported by July 10, 2020, a reported incidence of the 14-day COVID-19 cases, and having all available parameters were included in the study. Countries with ascertained and/or missing parameters were excluded from this study. A cross-continental comparison was also performed. To analyze the data, One-Way analysis of variance (One-Way ANOVA) followed by the Bonferroni test and Pearson correlation coefficient were conducted.

Results: In the final analysis, 137 countries were eligible. The median age, population aged over 65 years, female smoking rates, and life expectancy were positively correlated with the COVID-19 CFRs, while no significant correlation was found with diabetes prevalence, frequency of available handwashing centers, and number of hospital beds per 1,000 persons. The European continent had higher COVID-19 CFRs, while the Asian continent had higher cases/1 million population.

Conclusion: It is important to highlight the risk factors for mortality due to the novel coronavirus. This will help to anticipate healthcare needs and implement appropriate mitigation strategies, as well as to prioritize the most vulnerable individuals, thereby increasing their chances of survival from COVID-19.
\end{abstract}

Keywords: Case fatality rate, COVID-19, risk factors, continents, handwashing, smoking

ORCID IDs of the authors: E.K.P. 0000-0001-8099-8883; G.B. 0000-0001-5899-566X; K.N.B. 0000-0002-2785-0406; M.A. 0000-0003-4195-5797. 


\section{INTRODUCTION}

In December 2019, cases of an "unrecognized viral pneumonia" were detected among individuals working in or in contact with the local seafood market in Wuhan, China (1). The cause of this infection has been identified as a new subtype of coronavirus, referred to as Severe Acute Respiratory Syndrome-Coronavirus-2 (SARS-CoV-2), named Coronavirus disease (COVID-19) by the World Health Organization (WHO) (2). By the end of March 2020, the new coronavirus had spread rapidly from China to Europe, followed by a pandemic in the United States, which became the new global epicenter of the coronavirus $(3,4)$. In early March 2020, the Director-General of the WHO declared the COVID-19 as a pandemic (5). At that time, COVID-19 had spread to six continents and all the regions of the United Nations (6).

Countries had diverse responses to the COVID-19 pandemic. Many countries have implemented national regulations, such as quarantine, curfews, travel restrictions (7), and border closings, as mitigation strategies (8). In addition, personal protective measures have been taken, including the use of face masks and hand hygiene to reduce the spread of the coronavirus.

Worldwide, the pandemic has resulted in many deaths. According to currently available data, elderly indivduals, men, and those with underlying health diseases have been more affected by this contagious infection $(9,10)$.

The relationship between the COVID-19 infection and smoking has been examined, but remains controversial. While some studies have found an association between smoking and the disease severity and mortality, some studies have not. All of these prompted us to investigate whether there is a link between COVID-19 and smoking and whether gender plays a role in this difference.

We aimed to highlight the relationship between the sociodemographic and epidemiologic risk factors in comparison with COVID-19 case fatality rates (CFR). Furthermore, we conducted a comparison between population-based risk factors and COVID-19 CFR across the continents.

\section{METHODS}

\section{Study Design}

This cross-sectional study was conducted by examining data using the relational scanning model. The electronic dataset was extracted from the open-source database on July 10, 2020. This study was approved by the University of Health Sciences Turkey, Hamidiye Scientific Research Ethics Committee (approval number: 20/309).

\section{Data Collection}

The socio-demographic and COVID-19 data were obtained from the open-source websites, Our World in Data (https:// ourworldindata.org.), and the European Center for Disease Prevention and Control websites (https://github.com/owid/ covid19 data/tree/master/public/data), which are updated daily and include data on confirmed cases, deaths, and tests for all countries. The demographic and epidemiologic data that were identified as risk factors for COVID-19 CFRs were used. In the final analysis, 137 of the 215 affected countries were eligible for the study.

Abstracted data included the total number of cases per country, rate of confirmed cases per 1 million population, total population of the countries, population densities, median age, population rates over 65 and 70 years, diabetes prevalence, smoking rates among women and men, frequency of handwashing centers, number of hospital beds for 1,000 population, and life expectancy.

\section{Study Criteria}

Countries with at least 1,000 confirmed COVID-19 cases reported by July $10^{\text {th }}$, a reported incidence of the 14-day COVID-19 cases, and having all the available parameters were included in the study. Countries with ascertained and/or missing parameters were excluded from the study.

\section{Statistical Analysis}

All statistical analyses were performed using SPSS (IBM SPSS version 25) for Windows software. Percentage and frequency values for categorical variables and arithmetic mean ( \pm standard deviation) or median values for quantitative variables were used in the descriptive statistics of the data. The relationship between CFR and all other variables were determined using the Pearson correlation coefficient. Comparisons between continents were performed using a One-Way analysis of variance (One-Way ANOVA) followed by the Bonferroni post-hoc comparison test. In this study, a type I error rate of 0.05 , and p-value $<0.05$ were considered statistically significant.

\section{RESULTS}

We analyzed the risk factors that were identified in the sociodemographic and epidemiologic data for COVID-19 mortality. In our study, only 137 out of approximately 215 affected countries were selected for the analysis.

The total number of cases ( $p=0.344)$, rate of cases per one million population ( $p=0.501)$, total population of the country $(p=0.513)$, population density $(p=0.252)$, diabetes prevalence $(p=0.097)$, smoking rates among men $(p=0.641)$, frequency of available hand washing centers $(p=0.495)$, number of hospital beds per 1,000 population ( $p=0.395)$, and mortality rates were not significantly correlated with COVID-19 CFRs. Median age ( $p=0.001 ; r=0.272)$, population aged $>65$ years $(p<0.001 ; r=0.366)$, population aged $>70$ years $(p<0.001 ; r=0.378)$, and female smoking rate $(p<0.001$; $r=0.301$ ) were positively correlated with COVID-19 CFR. A very weak positive correlation was found between life expectancy $(p=0.011 ; r=0.219)$ and COVID-19 CFR (Table 1). 
A significant difference was determined between the COVID-19 CFRs and cases/one million ( $p=0.005)$, deaths/one million $(p<0.001)$. The continents of Asia and South America in cases/one million and Europe and South America in deaths/one million had higher values than other continents. The European continent had the highest COVID-19 CFR value (Table 2). In addition, the case fatality rates of the countries participating in our study are shown in Figure 1.

\section{Table 1. Socio-demographic risk factors and COVID-19 case fatality rates}

Total cases

Total cases (per million)

Population

Population density (people per sq. km of land area)

Aged $>65$ years ( $\%$ percent)

Aged $>70$ years (\% percent)

Diabetes prevalence (\% of population ages 20 to 79 )

Female smokers (\% of adults)

Male smokers (\% of adults)

Handwashing facilities (\% percent)

Hospital beds (per 1,000 population)

Life expectancy

${ }^{*} p<0.05$. COVID-19: Coronavirus disease
Case fatality rates

0.081

0.344

137

$-0.058$

0.501

137

0.056

0.513

137

$-0.099$

0.252

136

0.272

0.001

136

0.366

$<0.001$

136

0.378

$<0.001$

135

$-0.143$

0.097

136

0.301

0.001

111

$-0.045$

0.641

109

0.087

0.495

64

0.077

0.395

123

0.219

0.011

136 


\section{DISCUSSION}

In this study, we investigated the risk factors of the COVID-19 CFR according to demographic and epidemiologic characteristics. In reported studies to date, several risk factors have been associated with the disease mortality. The most important factors affecting the fatality rates were demographic characteristics such as age, smoking, and underlying health conditions $(9,11,12)$. This study revealed that age over 65 years and longer life expectancy were significantly correlated with increased mortality among

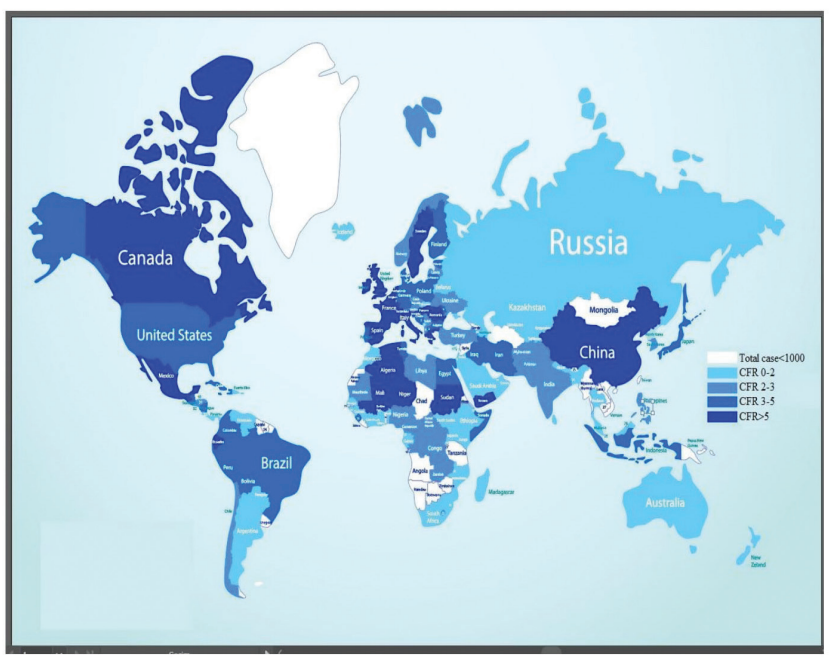

Figure 1. Case fatality rates of COVID-19 pandemic, July 10, 2020

CFR: Case fatality rates, COVID-19: Coronavirus disease

Table 2. Comparison of total cases, deaths, and CFRs between continents

\begin{tabular}{|c|c|c|c|c|c|}
\hline & Continents & Mean & SD & $\mathrm{F}$ & $p$ \\
\hline \multirow{3}{*}{ Total cases/1 M (per million) } & Asia & 3921.72 & 6925.53 & \multirow{3}{*}{3.846} & \multirow{3}{*}{0.005} \\
\hline & Europe & 2747.82 & 2048.63 & & \\
\hline & North America & 3007.26 & 3120.68 & & \\
\hline \multirow{4}{*}{ Total deaths/1 M (per million) } & Asia & 31.0 & 40.67 & \multirow{4}{*}{9.396} & \multirow{4}{*}{$<0.001$} \\
\hline & Europe & 163.79 & 212.57 & & \\
\hline & North America & 109.90 & 123.82 & & \\
\hline & South America & 174.94 & 149.38 & & \\
\hline \multirow[t]{3}{*}{ Case fatality rate } & Europe & 5.43 & 4.52 & \multirow[t]{3}{*}{4.616} & \multirow[t]{3}{*}{0.002} \\
\hline & North America & 3.75 & 3.07 & & \\
\hline & South America & 3.13 & 2.07 & & \\
\hline
\end{tabular}

COVID-19 patients across countries, which is consistent with most published data to date (13-15). This is explained by the fact that, as individuals age, they have concomitant diseases that could weaken their immune system, making them vulnerable to this disease $(14,16)$. This finding suggests that protective measures against COVID-19 should be implemented, especially in places such as nursing homes.

Remuzzi and Remuzzi (17) indicated that the mean age of patients who died from COVID-19 in Italy was 81 years, and two-thirds of them had underlying health conditions such as diabetes or were smokers. Since Italy is the country with one of the longest life expectancies and has an elderly population in Europe, it had the majority (77.4\%) of COVID-19 cases reported from Europe as of February 29 (6). As a result, Italy was the top affected country in Europe, followed by Spain, France, and Germany, and had the highest COVID-19 case-fatality rates in Europe. Consequently, these explain why the COVID-19 CFR of Europe was higher compared to that of other continents. This fact differs in China, which makes up only $12 \%$ of the elderly population in the Asian continent, explaining why the Asian continent lagged behind in the continental ranking in terms of deaths/one million population.

In the cross-continental comparison of cases and deaths per one million population, continental rankings have changed. Several reasons for this change include the differences in the sociodemographic structures between countries, such as age, life expectancy, population density, and accompanying diseases.

While the COVID-19 CFR is expected to be high in impoverished continents such as Africa, due to insufficient protective equipment, poor sources, and low number of physicians per

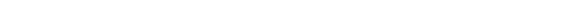


capita, we are facing low CFR results. Additionally, although the number of cases is high in India, the reported death rates are low. The reasons for the different or unreliable CFR results between countries that make it difficult to compare the CFRs can be listed as follows: definition of death due to COVID-19 varies between countries, different ways of measuring CFRs, insufficient number of tests, differences in the timing of tests and tracing the contacts, shortages of personal protective equipment, inadequate number of available intensive care beds, presence of different health systems, and political factors.

To our knowledge, no previous study has shown that diabetes has an independent predictive value for mortality, but a large number of studies have been conducted on the impact of diabetes on the disease progression and prognosis of COVID-19 patients (10). In addition, we have not found an association between diabetes prevalence and COVID-19 CFR.

Researchers have investigated smoking as one of the epidemiologic risk factors for COVID-19 disease $(15,18)$, but it is still controversial. In a meta-analysis, a statistically significant association was found between the risk of severe COVID-19 disease and death among smokers $(19,20)$. In the study of Zhang et al. (13), only $1.4 \%$ of patients were smokers, while in the study of Guan et al. (21), the rate of smokers was $12.6 \%$. When comparing the ratio of a small number of smokers to the proportion of smokers in the world, we are unable to draw a definitive relationship between the incidence or severity of COVID-19 and smoking status due to the lack of data (22). In our study, when we analyzed the association between smoking status and the COVID-19 CFRs in terms of gender, women smokers were more likely than men smokers to die of the coronavirus. Data from 137 countries suggest that this may be explained by the longer life expectancy of women compared to men. Even though these results differ from some earlier studies, compared with what was previously known, we currently do not have sufficient evidence to take into consideration the gender roles between smokers and the COVID-19 CFRs. To date, there is no convincing evidence demonstrating that hand hygiene can reduce the spread of SARSCoV-2 (23). Although handwashing plays a crucial protective role in deterring epidemic transmission, the significance of reducing the spread of COVID-19 infection is effective when combined with strategies that include simple preventative measures such as face mask use, disinfection, social distancing, and disease awareness rather than only emphasizing the importance of hand hygiene (24). Our study results are also in line with previous studies.

\section{Study Limitations}

The strength of our study is that it covers countries with over 1,000 reported COVID-19 cases, which enables stable analysis. However, this study has several limitations. The first is that the epidemiologic data was collected using a retrospective design. The second is the under-reporting or lack of additional information, such as medications, body mass index, behavioral changes, and gender-checked smoking status, all of which may influence the published results. Hence, we recommend further detailed research to highlight these knowledge gaps regarding the COVID-19 pandemic.

\section{CONCLUSION}

In summary, this article highlighted the risk factors affecting COVID-19 CFRs across countries, which will help to determine appropriate mitigation strategies, prioritize the most vulnerable individuals, and increase their chances of survival from the COVID-19.

Ethics Committee Approval: This study was approved by the University of Health Sciences Turkey, Hamidiye Scientific Research Ethics Committee (approval number: 20/309).

Informed Consent: Cross-sectional study.

Peer-review: Externally peer-reviewed.

Author Contributions: Concept - E.K.P., G.B., M.A.; Design - E.K.P., G.B., K.N.B.; Data Collection and/or Processing - G.B., K.N.B.; Analysis and/ or Interpretation - G.B., K.N.B.; Literature Search - E.K.P., G.B., M.A.; Writing Manuscript - E.K.P., M.A

Conflict of Interest: The authors have no conflict of interest to declare.

Financial Disclosure: The authors declared that this study has received no financial support.

\section{REFERENCES}

1. National Health Commission of the People's Republic of China. New coronavirus cases rise to 571 in Chinese mainland. Available from: http:// en.nhc.gov.cn/202001/23/c_76004.htm

2. He F, Deng Y, Li W. Coronavirus disease 2019: What we know? J Med Virol 2020; 92: 719-25.

3. World Health Organization. Novel coronavirus situation report-2. January 22, 2020. Available from: https://www.who.int/docs/default-source/ coronaviruse/situation-reports/20200122-sitrep-2-2019-ncov.pdf

4. European Centre for Disease Prevention and Control. Geographical distribution of 2019nCov cases. Available from: https://www. ecdc. europa.eu/en/geographical-distribution2019-ncov-cases.

5. World Health Organization. Director-General's remarks at the media briefing on 2019-nCoV on 11 February 2020. Availabl from: https:// www.who.int/director-general/speeches/detail/who-director-general-sremarks-at-the-media-briefing-on-2019-ncov-on-11-february-2020

6. Shultz JM, Perlin A, Saltzman RG, Espinel Z, Galea S. Pandemic March: 2019 Coronavirus Disease's First Wave Circumnavigates the Globe. Disaster Med Public Health Prep 2020;1-5.

7. Juliana Kaplan LF. A third of the global population is on coronavirus lockdown-here's our constantly updated list of countries and restrictions. Business Insider; 2020. Available from: https://www.businessinsider.in/ international/news/a-third-of-the-global-population-is-on-coronaviruslockdown-x2014-hereaposs-our-constantly-updated-list-of-countriesand-restrictions/slidelist/75208623.cms

8. Salcedo A, Yar S, Cherelus G. Coronavirus Travel Restrictions, Across the Globe. The New York Times; 2020. Available from: https://www.nytimes. com/article/coronavirus-travel-restrictions.html

9. Yi Y, Lagniton P, Ye S, Li E, Xu RH. COVID-19: what has been learned and to be learned about the novel coronavirus disease. Int J Biol Sci 2020; 16: 1753-66.

10. Tadic M, Cuspidi C, Sala C. COVID-19 and diabetes: Is there enough evidence? J Clin Hypertens (Greenwich) 2020; 22: 943-8.

11. Du RH, Liang LR, Yang CQ, Wang W, Cao TZ, Li M, et al. Predictors of mortality for patients with COVID-19 pneumonia caused by SARS-CoV-2: a prospective cohort study. Eur Respir J 2020; 55: 2000524.

12. Onder G, Rezza G, Brusaferro $S$. Case-fatality rate and characteristics of patients dying in relation to COVID-19 in Italy. JAMA 2020; 323: 1775-6. 
13. Zhang JJ, Dong $X$, Cao $Y Y$, Yuan $Y D$, Yang YB, Yan $Y Q$, et al. Clinical characteristics of 140 patients infected with SARS-CoV-2 in Wuhan, China. Allergy 2020; 75: 1730-41.

14. Wang D, Hu B, Hu C, Zhu F, Liu X, Zhang J, et al. Clinical characteristics of 138 hospitalized patients with 2019 Novel Coronavirus-Infected pneumonia in Wuhan, China. JAMA 2020; 323: 1061-9.

15. Liu W, Tao ZW, Wang L, Yuan ML, Liu K, Zhou L, et al. Analysis of factors associated with disease outcomes in hospitalized patients with 2019 novel coronavirus disease. Chin Med J 2020; 133: 1032-8.

16. Wang L, He W, Yu X, Hu D, Bao M, Liu H. et al. Coronavirus disease 2019 in elderly patients: Characteristics and prognostic factors based on 4-week follow-up. J Infect 2020; 80: 639-45.

17. Remuzzi A, Remuzzi G. COVID-19 and Italy: what next? Lancet 2020; 395: 1225-8.

18. Vardavas Cl, Nikitara K. COVID-19 and smoking: A systematic review of the evidence. Tob Induc Dis 2020; 18: 20.

19. Zhao Q, Meng M, Kumar R, Wu Y, Huang J, Lian N, et al. The impact of COPD and smoking history on the severity of COVID-19: A systemic review and metaanalysis. J Med Virol 2020; doi: 10.1002/jmv.25889 [Online ahead of print].

20. Zheng Z, Peng F, Xu B, Zhao J, Liu H, Peng J, et al. Risk factors of critical \& mortal COVID-19 cases: A systematic literature review and meta-analysis. J Infect 2020; 81: e16-25.

21. Guan WJ, Ni ZY, Hu Y, Liang WH, Ou CQ, He JX, et al. China Medical Treatment Expert Group for Covid-19 clinical characteristics of Coronavirus disease 2019 in China. N Eng J Med 2020; 382: 1708-20.

22. Cai H. Sex difference and smoking predisposition in patients with COVID-19. Lancet Respir Med 2020; 8: e20.

23. West R, Michie $S$, Rubin GJ, Amlôt R. Applying principles of behaviour change to reduce SARS-CoV-2 transmission. Nat Hum Behav 2020; 4: 451-9.

24. Yang C. Does hand hygiene reduce SARS-CoV-2 transmission? Graefe's archive for clinical and experimental ophthalmology = Albrecht von Graefes Archiv fur klinische und experimentelle. Ophthalmologie 2020; 258: 1133-4. 